\title{
Numerical investigation of planar shock wave impinging on spherical gas bubble with different densities
}

Cite as: Phys. Fluids 31, 056101 (2019); https://doi.org/10.1063/1.5092317

Submitted: 10 February 2019 . Accepted: 21 April 2019 . Published Online: 06 May 2019

Yuejin Zhu (朱跃进), Zhiwei Yang (杨志伟), Kai Hong Luo (罗开红) (D), Jianfeng Pan (潘剑锋), and Zhenhua Pan (潘振华)

\section{ARTICLES YOU MAY BE INTERESTED IN}

On the role of rarefaction/compression waves in Richtmyer-Meshkov instability with reshock

Physics of Fluids 31, 054102 (2019); https://doi.org/10.1063/1.5083796

Hypersonic aerodynamic heating over a flared cone with wavy wall

Physics of Fluids 31, 051702 (2019); https://doi.org/10.1063/1.5098543

A numerical study on bubble dynamics in sinusoidal channels

Physics of Fluids 31, 052103 (2019); https://doi.org/10.1063/1.5092870

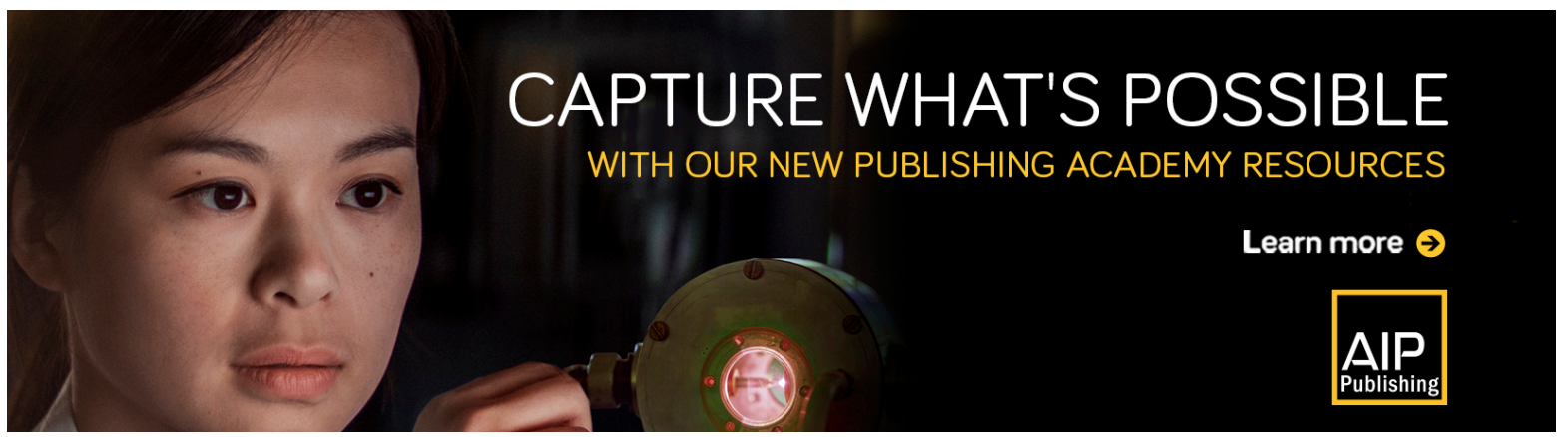




\title{
Numerical investigation of planar shock wave impinging on spherical gas bubble with different densities
}

\author{
Cite as: Phys. Fluids 31, 056101 (2019); doi: 10.1063/1.5092317 \\ Submitted: 10 February 2019 - Accepted: 21 April 2019 • \\ Published Online: 6 May 2019
}

Yuejin Zhu (朱跃进), ${ }^{1,2}$ Zhiwei Yang (杨志伟), ${ }^{7}$ Kai Hong Luo (罗开红), ${ }^{2, a)}$ (D) Jianfeng Pan (潘剑锋), and Zhenhua Pan (潘振华) $)^{7,6)}$

\author{
AFFILIATIONS \\ 'School of Energy and Power Engineering, Jiangsu University, Zhenjiang 212013, China \\ ${ }^{2}$ Department of Mechanical Engineering, University College London, Torrington Place, London WC1E 7JE, United Kingdom
}

a) Electronic mail: k.luo@ucl.ac.uk

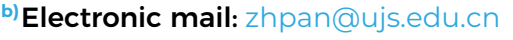

\begin{abstract}
The interaction between a planar shock wave and a spherical gas bubble containing sulfur hexafluoride, Refrigerant-22, neon, or helium is studied numerically. Influences of the Atwood number (At) on the evolution of the shock wave and gas bubble are clarified by using highresolution computational simulations. The results show that the difference in the physical properties between the ambient air and the gas bubble has a significant influence on the evolution of wave pattern and bubble deformation. For the fast/slow configuration (At $>0$ ) in the present study (At $=0.67$ and 0.51 ), the incident shock focuses near the interior right interface to form an outward jet. Besides, the mixedness, average vorticity, and the absolute value of circulation all increase as the Atwood number increases. By contrast, for the slow/fast configuration $($ At $<0)$ with $\mathrm{At}=-0.19$ and -0.76 , the rotational directions of the vorticities formed at the same position are reversed compared with those in the fast/slow configuration, which induces an inward air jet to impact on the gas bubble from the outside. In addition, the mixedness, average vorticity, and the absolute value of circulation all increase as the Atwood number decreases. Nevertheless, regardless of At $>0$ or At $<0$, the effective volume of the gas bubble basically decreases when the Atwood number decreases. Hence, on the whole, the Atwood number has a nonmonotonic influence on the evolution of effective volume of gas bubble, mixedness, average vorticity, and circulation simultaneously.
\end{abstract}

Published under license by AIP Publishing. https://doi.org/10.1063/1.5092317

\section{INTRODUCTION}

During the interaction between a shock wave and a gas bubble, vorticities will deposit on the bubble interface owing to the baroclinic effect (misalignment between the pressure and density gradients), and they can accelerate the mixing between different gases. This phenomenon mainly involves the RichtmyerMeshkov instability (RMI), which frequently occurs in natural and manmade activities. ${ }^{1-3}$ Therefore, research in this area is of great significance.

Many relevant experimental and numerical studies have been conducted and reported in the past. In 1987, Haas and Sturtevant ${ }^{4}$ used the shadowgraphy technology in their experiment to exhibit the light (helium, He) and heavy [Refrigerant-22 (R22), a fluorocarbon (CHClF2)] bubbles' deformation processes; however, only one photograph was taken during each run. Then, Jacobs ${ }^{5,6}$ experimentally studied the interactions between cylindrical gas bubbles [light $(\mathrm{He})$ and heavy (sulfur hexafluoride, $\left.\mathrm{SF}_{6}\right)$ ] and a weak shock wave by adopting the planar laser induced fluorescence (PLIF) technology, and high quality flow visualization was obtained. By means of the high-speed shadowgraph system, Layes et al. ${ }^{7-9}$ investigated the gas bubble evolution of different gases [He, nitrogen $\left(\mathrm{N}_{2}\right)$ and krypton $(\mathrm{Kr})]$, which were impacted by a planar shock wave. The obtained high quality experimental results could be used for validation of other numerical and theoretical studies. During the same period, the interaction between a shock wave and a spherical bubble in divergent shock-refraction geometry was studied using the shock tube experiments by Ranjan et al. ${ }^{10} \mathrm{~A}$ helium bubble in ambient air 
or nitrogen was considered, and the experimental planar laser diagnostics clearly resolved the features of the flow field. Actually, the relationship between the gas bubble and the ambient gas is generally represented by a dimensionless parameter called the Atwood number, At $=\left(\rho_{2}-\rho_{1}\right) /\left(\rho_{2}+\rho_{1}\right)$, where $\rho_{1}$ and $\rho_{2}$ are the densities of the ambient and the bubble gases, respectively. To reveal the influence of the Atwood number on the shock-bubble interaction, two values of the Atwood number ( $\mathrm{At}=0.17$ and 0.68 ) were investigated experimentally by Haehn et al. ${ }^{11}$ in 2012 . The results showed that a secondary vortex ring appeared immediately after reshock for the low Atwood number case. In the same year, $\mathrm{Si}$ et al. ${ }^{12}$ performed a number of experimental sets of $\mathrm{He}$ and $\mathrm{SF}_{6}$ bubbles surrounded by air by using the high-speed Schlieren photography, and the differences between the two cases were studied.

In addition to the aforementioned experimental studies, Zabusky and Zeng ${ }^{13}$ simulated with the Euler equations planar shocks interacting with an Refrigerant-12 (R12) spherical bubble, and the collapsing shock cavity caused an expelled weak jet which was found at a low Mach number, but the "vortical projectiles" appear on the downstream side of the bubble at higher Mach numbers. Then, Bagabir and Drikakis ${ }^{14}$ numerically investigated the incident shock Mach number effects on the interaction of a shock wave with a bubble, which revealed the additional gas dynamic features with the increasing shock Mach numbers. Niederhaus et al. ${ }^{15}$ conducted a series of three-dimensional Euler simulations to analyze the processes of shock-bubble interaction, and the influences of the Atwood number $(-0.8<$ At $<0.7)$ were investigated. Remarkable results from the simulations were obtained when At $>0.5$; besides, a new mathematical model for velocity circulation was proposed. Then, Zhai et al. ${ }^{16}$ numerically studied the interaction of a planar shock wave with a spherical gas bubble which was filled with different gases $\left(\mathrm{SF}_{6}\right.$ and $\left.\mathrm{He}\right)$ by using the VAS2D code, and the detailed flow field structures including the evolution of bubble interfaces and waves were obtained. Subsequently, Zou et al. ${ }^{17}$ numerically studied the evolution of the heavy bubbles with different gases $\left(\mathrm{SF}_{6}, \mathrm{R} 22\right.$, $\mathrm{R} 12$, and $\mathrm{Kr}$ ) in the shock accelerated flow, and the influences of the different gases on the shock wave focusing and jet formation were investigated. Meanwhile, Georgievskiy et al. ${ }^{18}$ carried out a numerical research on the interaction of a shock wave with spherical bubbles of light or heavy gases by using the axisymmetric Euler equations. A computational parametric study for different At $(-0.54$ $\leq \mathrm{At} \leq 0.5$ ), shock strengths, and bubble geometries was performed. The results showed that the shock focusing pattern was governed by the Atwood number, Mach number, and the bubble shape simultaneously. The authors ${ }^{19-21}$ also numerically investigated the deformation and instability of $\mathrm{He}$ and $\mathrm{SF}_{6}$ bubbles, and the formation and evolution of vortex rings, shock wave, and the influences of initial parameters (such as the incident shock wave strengths) were analyzed in detail. Recently, the interaction between a planar shock wave and square and triangular bubbles containing $\mathrm{SF}_{6}, \mathrm{He}$, argon (Ar), or carbon dioxide $\left(\mathrm{CO}_{2}\right)$ was numerically studied by Igra and Igra. ${ }^{22}$ The results showed that different wave patterns and pressure distribution inside the bubbles were developed owing to the large physical differences (such as the acoustic impedance) between these gases.

In summary, a lot of experimental and numerical studies on the interactions between the shock wave and gas bubble have been carried out. However, it should be noted that previous studies mainly concentrate on the interaction between a shock wave and one or two kinds of gas bubbles. Furthermore, even when the shock waves interacting with bubbles filled with various kinds of gases are considered, the influences of the Atwood number on the shock wave-bubble interaction have not been analyzed thoroughly, especially from the viewpoint of quantitative analysis. Therefore, in the present study, by means of numerical simulation based on a high accuracy method and a high grid resolution, the influences of the Atwood number at different values $[\mathrm{At}=0.67,0.51,-0.19$ and -0.76 , corresponding to $\mathrm{SF}_{6}, \mathrm{R} 22$, neon $(\mathrm{Ne})$, and $\mathrm{He}$ gases in ambient air, respectively] on the interaction between a shock wave and a gas bubble are discussed qualitatively and quantitatively. To be specific, the evolution of the shock wave and the gas bubble, and the time-dependent integral properties all need to be analyzed to reveal the influences of the Atwood number.

\section{NUMERICAL METHOD AND SETUP}

\section{A. Numerical method}

The two-dimensional Navier-Stokes (NS) equations are expressed as

$$
\frac{\partial U}{\partial t}+\frac{\partial F}{\partial x}+\frac{\partial G}{\partial r}+W=0
$$

where

$$
\begin{aligned}
& U=\left[\begin{array}{l}
\rho \\
\rho u \\
\rho v \\
E \\
\rho Y
\end{array}\right], \quad F=\left[\begin{array}{c}
\rho u \\
\rho u^{2}+p-\tau_{x x} \\
\rho u v-\tau_{r x} \\
(E+p) u-k \partial T / \partial x-u \tau_{x x}-v \tau_{r x} \\
\rho u Y-D \rho \partial Y / \partial x
\end{array}\right], \\
& G=\left[\begin{array}{c}
\rho v \\
\rho u v-\tau_{x r} \\
\rho v^{2}+p-\tau_{r r} \\
(E+p) v-k \partial T / \partial y-u \tau_{x r}-v \tau_{r r} \\
\rho v Y-D \rho \partial Y / \partial r
\end{array}\right], \quad W=\frac{v}{r}\left[\begin{array}{l}
\rho \\
\rho v \\
\rho+E \\
\rho Y
\end{array}\right] .
\end{aligned}
$$

In the above equations, $\rho$ is the density, $u$ and $v$ are the velocities in $x$ and $r$ directions, respectively, $E$ is the unit volume total energy, $Y$ is the mass fraction of the ambient gas, and $P$ is the pressure. $\tau_{x x}$, $\tau_{x r}, \tau_{r x}$, and $\tau_{r r}$ are the viscous stresses, which can be written as $\tau_{x x}$ $=4 / 3(\mu \partial u / \partial x)-2 / 3(\mu \partial v / \partial r), \tau_{x r}=\tau_{r x}=\mu(\partial u / \partial x+\partial v / \partial r)$, and $\tau_{r r}$ $=4 / 3(\mu \partial v / \partial r)-2 / 3(\mu \partial u / \partial x)$. The thermal conductivity $k$, the diffusion coefficient $D$, and the kinematic viscosity are given according to the ambient gas properties. The values are set to $k=2.34 \times 10^{-2} \mathrm{~J}$ $\mathrm{m}^{-1} \mathrm{~K}^{-1} \mathrm{~s}^{-1}, \mu=1.86 \times 10^{-5} \mathrm{~kg} \mathrm{~m}^{-1} \mathrm{~s}^{-1}$, and $D=1.73 \times 10^{-5} \mathrm{~m}^{-2} \mathrm{~s}^{-1}$, respectively.

To avoid unphysical oscillations for the computational results, a global Lax-Friedrich's flux splitting combined with a ninth-order weighted essentially nonoscillatory (WENO) scheme ${ }^{23}$ is adopted to discretize the inviscid fluxes. A tenth-order central difference scheme is employed to solve the viscous fluxes. Moreover, a 


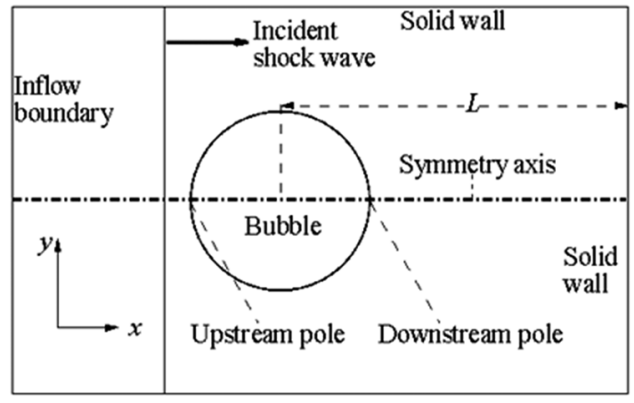

FIG. 1. Computational domain and initial conditions.

third-order Runge-Kutta method is used to integrate the equations in time marching.

\section{B. Computational setup}

The computational domain and the initial conditions are shown in Fig. 1. A spherical gas bubble surrounded by air located at the symmetry axis of the flow field. The symbol $L$ represents the end wall distance, which is the distance between the bubble center and the end wall. The distance between the gas bubble center and the left $x$ direction boundary is chosen sufficiently large to avoid artifacts due to shock reflections and expansions. ${ }^{24}$ The initial pressure $P_{0}$ is $1 \mathrm{~atm}$, and the incident shock wave first propagates from left to right to impinge on the bubble, then it reflects from the right wall to impinge on the distorted bubble again.

The no-slip wall conditions are enforced on the solid surfaces of the computational boundary, while the inflow condition is enforced on the $x=0 \mathrm{~mm}$ surface. To reduce the calculation, half the flow field is utilized as the computation domain, and thus, an axial symmetrical boundary condition is used. The uniform mesh size $0.1 \mathrm{~mm}$ is adopted in the present study, which is sufficient to ensure the computational accuracy and minimize the computation cost simultaneously. ${ }^{20,21}$

\section{RESULTS AND DISCUSSION}

\section{A. Numerical validation}

To demonstrate the reliability of the present numerical method and model, numerical results of the interaction between the planar shock wave and the spherical gas bubble with different densities $\left(\mathrm{SF}_{6}, \mathrm{Ne}\right.$, and $\left.\mathrm{He}\right)$ are first compared with the experimental results of $\mathrm{Si}^{12}$ and Layes. ${ }^{9}$ Table I presents the initial conditions of the three different experimental cases, and the comparisons between experimental Schlieren images and computational Schlieren results at the selected time instants are shown in Figs. 2-4.

Figure 2 shows the comparisons between the experimental and the numerical simulation results during the shock- $\mathrm{SF}_{6}$ bubble interaction, where Fig. 2(a) corresponds to the interaction between the initial $\mathrm{SF}_{6}$ bubble and incident shock wave, Fig. 2(b) the focusing of refracted shock waves inside of the bubble, and Fig. 2(c) the interaction between the distorted $\mathrm{SF}_{6}$ bubble and the reflected shock wave.
TABLE I. Initial conditions used in the three different experimental cases including the incident planar shock Mach number $\mathrm{Ma}$, the initial bubble radius $R_{0}(\mathrm{~mm})$, the end wall distance $L(\mathrm{~mm})$, and the bubble gas density $\rho\left(\mathrm{kg} \mathrm{m}^{-3}\right)$. Note that $L \rightarrow \infty$ denotes the case without reshock.

\begin{tabular}{|c|c|c|c|c|c|c|}
\hline $\begin{array}{l}\text { Bubble } \\
\text { gas }\end{array}$ & References & $\mathrm{Ma}$ & $\begin{array}{c}R_{0} \\
(\mathrm{~mm})\end{array}$ & $\begin{array}{c}L \\
(\mathrm{~mm})\end{array}$ & $\begin{array}{c}\rho \\
\left(\mathrm{kg} \mathrm{m}^{-3}\right)\end{array}$ & $\begin{array}{c}\text { Surrounding } \\
\text { gas }\end{array}$ \\
\hline $\mathrm{SF}_{6}$ & Si et al. ${ }^{12}$ & 1.21 & 16.25 & 79 & 6.030 & Air \\
\hline $\mathrm{Ne}$ & Layes et al. ${ }^{9}$ & 1.62 & 21.00 & $\infty$ & 0.802 & Air \\
\hline $\mathrm{He}$ & Si et al. ${ }^{12}$ & 1.23 & 14.95 & 64 & 0.160 & Air \\
\hline
\end{tabular}

The results suggest that the $\mathrm{SF}_{6}$ bubble are compressed into an elliptical shape induced by the incident shock wave in Fig. 2(a), and the refracted shock within the $\mathrm{SF}_{6}$ bubble propagates more slowly than the outside incident shock. Subsequently, the shock focusing occurs in Fig. 2(b), and a local high pressure zone emerges near the downstream pole which accelerates the right interface of the $\mathrm{SF}_{6}$ bubble. In Fig. 2(c), a long downstream $\mathrm{SF}_{6}$ jet emerges, and the reflected shock wave impinges on the distorted bubble again. Moreover, a large number of small vortices form throughout the bubble interface at this time instant.

Figure 3 presents the comparisons between the experimental and the numerical simulation results during the shock-Ne bubble interaction, where Fig. 3(a) shows the initial interaction of the $\mathrm{Ne}$ bubble with the incident shock wave, and Figs. 3(b) and 3(c) show the incident shock wave has completely passed through the bubble. The results indicate that the left interface of the Ne bubble is flattened by the incident shock in Fig. 3(a), and the refracted shock within the bubble propagates slightly faster than the outside incident shock. Then, the entire Ne bubble has been elongated significantly when the incident shock wave has just passed through the bubble in Fig. 3(b). As there is no reshock in this case, the $\mathrm{Ne}$ bubble evolves into the shape of an elongated squash and some small vortices are generated at the top and bottom of the bubble in Fig. 3(c).

Figure 4 exhibits the comparisons between the experimental and the numerical simulation results during the shock-He bubble interaction, where Fig. 4(a) presents the interaction of the He bubble with the incident shock wave, Fig. 4(b) the incident shock wave completely passing through the bubble, and Fig. 4(c) the interaction between the distorted $\mathrm{He}$ bubble and the reflected shock wave. The results illustrate that the left interface of the bubble is flattened by incident shock in Fig. 4(a), and the refracted shock within the bubble propagates significantly faster than the outside incident shock. Subsequently, the He bubble undergoes a reversal and the rear interface of the bubble gradually catches up with the front in Fig. 4(b). It should be noted that the experimental Schlieren result represents the three-dimensional projection of light, but the numerical result is obtained from two-dimensional simulation, and thus, the distorted He bubble in the experimental result seems larger than that in the numerical result. This result presents similarities with those reported by Layes and Metayer ${ }^{8}$ and Zhai et al. ${ }^{16}$ When the reflected shock from the end wall interacts with the distorted $\mathrm{He}$ bubble again in Fig. 4(c), the bubble evolves into the shape of a kidney and a smaller vortex ring emerges at the right interface of bubble. 

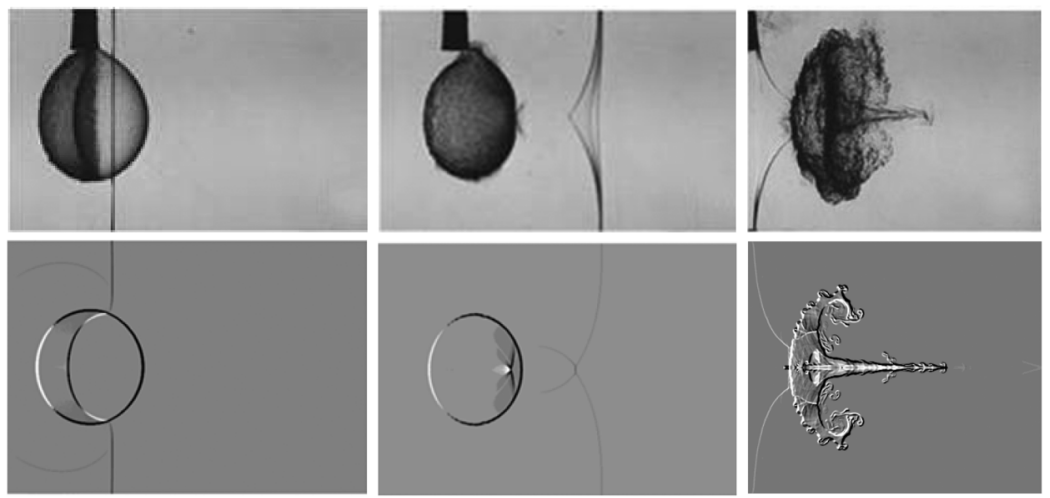

(a) $t=68.6 \mu \mathrm{s}$
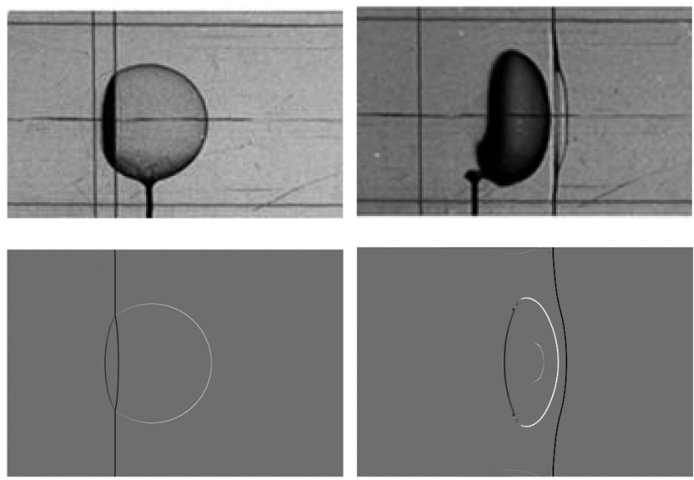

(a) $t=16.2 \mu \mathrm{s}$ (b) $t=140.5 \mu \mathrm{s}$

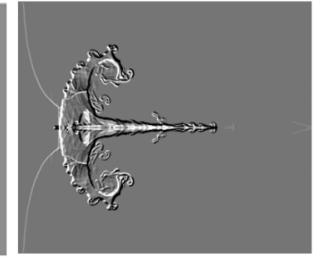

(c) $t=495.9 \mu \mathrm{s}$
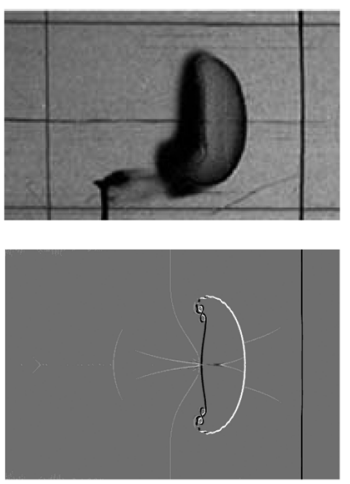

(c) $t=162.0 \mu \mathrm{s}$
FIG. 2. Comparisons of $\mathrm{SF}_{6}$ bubble between the experimental $^{12}$ (top) and the present computational (bottom) results.
FIG. 3. Comparisons of Ne bubble between the experimental $^{9}$ (top) and the present computational (bottom) results.
From Figs. 2-4, the good qualitative agreements between the experimental and the computational results have been obtained in the three cases, which indicate that the present numerical method and model are reliable in a wide range of bubble gas densities (i.e., different values of the Atwood number).

\section{B. Visualized flow fields}

As aforementioned, distinct evolution of the spherical gas bubble with different densities will emerge under the incident shock and reshock conditions, and the related complicated phenomena are
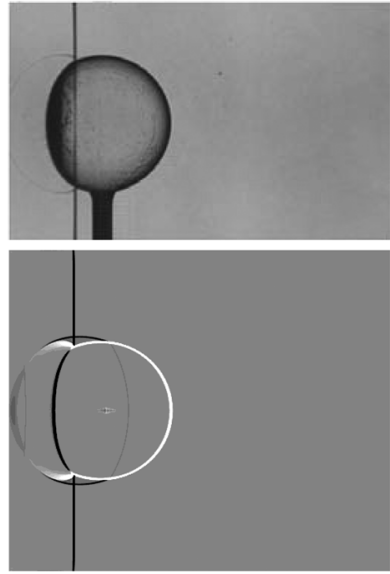

(a) $t=34.6 \mu \mathrm{s}$
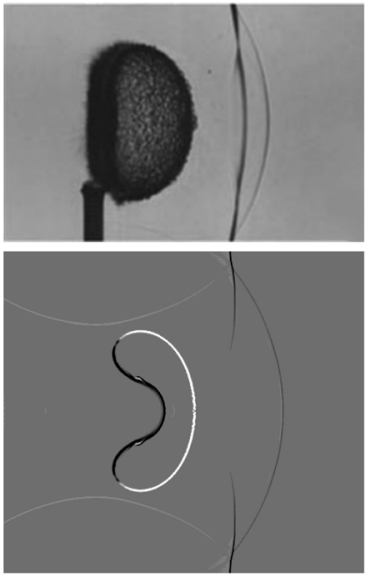

(b) $t=125.7 \mu \mathrm{s}$


(c) $t=297.6 \mu \mathrm{s}$
FIG. 4. Comparisons of He bubble between the experimental $^{12}$ (top) and computational (bottom) results. 
TABLE II. Initial conditions used in the four cases.

\begin{tabular}{lccccccc}
\hline \hline $\begin{array}{l}\text { Bubble } \\
\text { gas }\end{array}$ & $\mathrm{Ma}$ & $\begin{array}{c}R_{0} \\
(\mathrm{~mm})\end{array}$ & $\begin{array}{c}L \\
(\mathrm{~mm})\end{array}$ & $\begin{array}{c}\rho \\
\left(\mathrm{kg} \mathrm{m}^{-3}\right)\end{array}$ & $\begin{array}{c}\text { Acoustic impedance } \\
\left(\mathrm{kg} \mathrm{m}^{-2} \mathrm{~s}^{-1}\right)\end{array}$ & $\begin{array}{c}\text { Atwood number } \\
(\mathrm{At})\end{array}$ & $\begin{array}{c}\text { Surrounding } \\
\text { gas }\end{array}$ \\
\hline $\mathrm{SF}_{6}$ & 1.21 & 16.25 & 79 & 6.030 & 822.1 & 0.67 & Air \\
$\mathrm{R} 22$ & 1.21 & 16.25 & 79 & 3.690 & 635.8 & 0.51 & Air \\
$\mathrm{Ne}$ & 1.21 & 16.25 & 79 & 0.802 & 362.8 & -0.19 & Air \\
$\mathrm{He}$ & 1.21 & 16.25 & 79 & 0.160 & 162.1 & -0.76 & Air \\
\hline \hline
\end{tabular}

worthy of detailed studies. Hence, four cases with different values of the Atwood number under the incident and reshock conditions have been designed and simulated, and the computational size is $250 \mathrm{~mm}$ $\times 35 \mathrm{~mm}$. Table II gives the different initial conditions of the four cases, where the acoustic impedance is obtained by $Z=\rho c$ ( $\rho$ is the density and $c$ is the speed of sound), which indicates the resistance that a pressure wave needs to overcome when it propagates in a certain medium. Spherical gas bubbles with four typical different gases are considered in Table II, where the Atwood number At $<0$, At $\approx 0$ and At $>0$ mean the density of the gas bubble is heavier, similar, and lighter than the density of surrounding air, respectively, and the values vary from a large negative value to a large positive value which cover a wide range.

For the $\mathrm{SF}_{6}$ bubble case $(\mathrm{At}=0.67)$, Fig. 5 shows the evolution of waves and $\mathrm{SF}_{6}$ bubble at different time instants. Due to the larger acoustic impedance, the propagation velocity of the shock wave in the $\mathrm{SF}_{6}$ bubble is smaller than that in air. Therefore, the refracted shock wave (TS) inside the bubble is far behind the incident shock wave (IS) outside the bubble in Fig. 5(a). When the IS propagates around the $\mathrm{SF}_{6}$ bubble, a diffracted shock wave (DS) and an incident diffracted shock wave (DTS) emerge. A closer look shows that an undisturbed zone (UZ) is formed by the TS, DTS, and the right interface. As shown in Fig. 5(b), the DS waves outside the bubble first collide near the downstream pole of the bubble, and then the DTS waves inside the bubble will also collide near the downstream pole of the bubble. During this process, the TS and DTS converge continuously and their intensities continue increasing. Meanwhile, the area of UZ continues to shrink. As the TS and DTS get closer with time, the UZ will disappear eventually and the shock focusing occurs near the interior right interface, as shown in Fig. 5(c). In Fig. 5(d), the expanding shock wave (ES) generated by shock wave focusing impinges on the right interface of the bubble and leads to the formation of a $\mathrm{SF}_{6}$ jet (J). After passing through the right interface of the bubble, the ES continues to propagate to the downstream. At $t=443.2 \mu \mathrm{s}$ in Fig. 5(e), the reflected wave (RS) generated by the IS impacting the right end wall interacts with the significantly distorted $\mathrm{SF}_{6}$ bubble again, and a pair of large vorticities emerges up and down the $\mathrm{SF}_{6}$ bubble interface. When the RS propagates the distorted $\mathrm{SF}_{6}$ bubble completely in Fig. $5(\mathrm{f})$, the $\mathrm{SF}_{6}$ bubble is further deformed, and more vortices form on the surface of the bubble and jet. Actually, take the upper half of the $\mathrm{SF}_{6}$ bubble as an example, vorticities with clockwise direction emerge due to the impingement of the IS [as shown in Fig. 5(d)]. Whereas, owing to the impingement of the RS, new vorticities with counterclockwise direction emerge even though the clockwise vorticities exist [as shown in Fig. 5(f)]. Thus, the bubble interface becomes more complicated under the reshock conditions.

Considering the gas density and acoustic impedance of R22 are both smaller than those of $\mathrm{SF}_{6}$, there should be some differences in the evolution of waves and the R22 bubble. In order to study these differences in detail, Fig. 6 gives the Schlieren pictures of several typical time instants during the interaction between the shock waves and the R22 bubble. Compared with Fig. 5, the refracted shock



(a) $t=112.8 \mu \mathrm{s}$

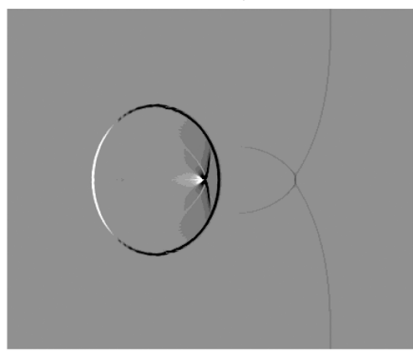

(c) $t=141.3 \mu \mathrm{s}$



(e) $t=443.2 \mu \mathrm{s}$



(b) $t=121.7 \mu \mathrm{s}$

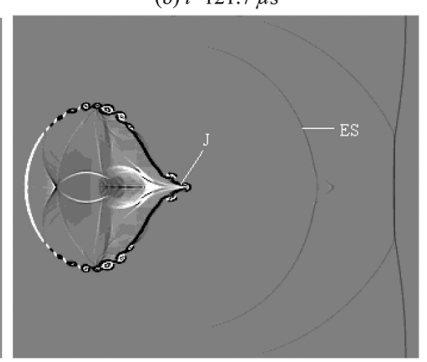

(d) $t=219.5 \mu \mathrm{s}$

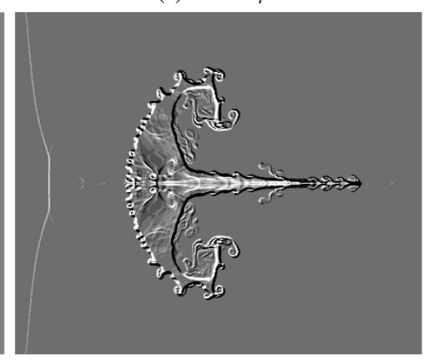

(f) $t=534.1 \mu \mathrm{s}$
FIG. 5. Evolution of the waves and the $\mathrm{SF}_{6}$ gas bubble. 


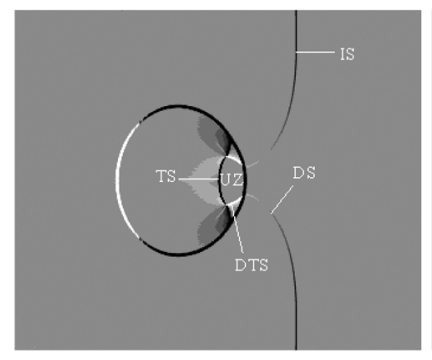

(a) $t=114.1 \mu \mathrm{s}$

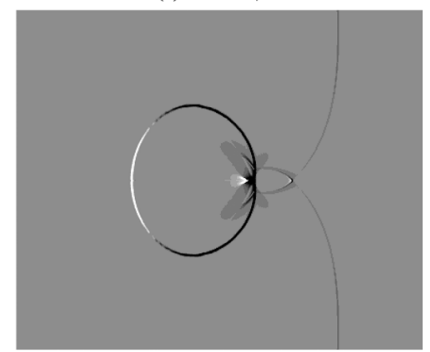

(c) $t=129.1 \mu \mathrm{s}$

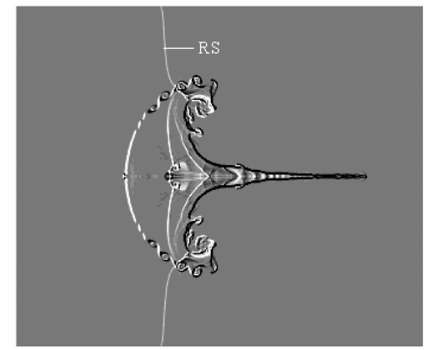

(e) $t=402.3 \mu \mathrm{s}$

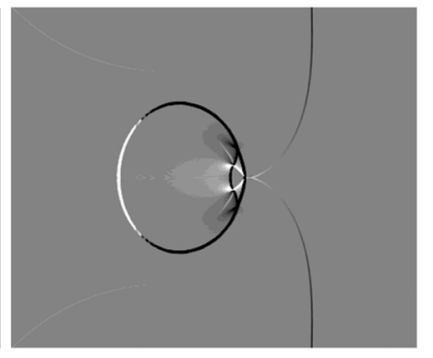

(b) $t=122.1 \mu \mathrm{s}$

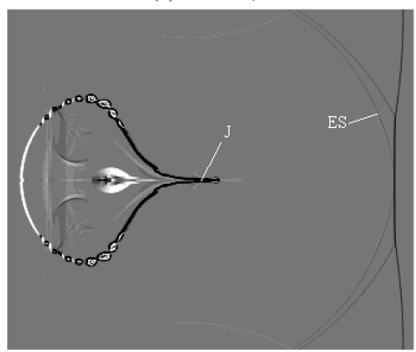

(d) $t=230.5 \mu \mathrm{s}$

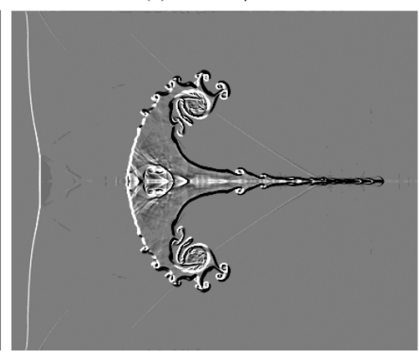

(f) $t=466.8 \mu \mathrm{s}$
FIG. 6. Evolution of the waves and the R22 gas bubble.

wave (TS) and incident diffraction shock wave (DTS) inside the bubble can also be seen clearly, and an undisturbed zone (UZ) is also formed by the TS, DTS, and the right interface. However, due to the acoustic impedance of $\mathrm{R} 22$ is smaller than that of $\mathrm{SF}_{6}$, the shock waves move faster in the $\mathrm{R} 22$ bubble than those in the $\mathrm{SF}_{6}$ bubble, and so the size of UZ in R22 bubble is also smaller than that in the $\mathrm{SF}_{6}$ bubble, as shown in Figs. 6(a) and 6(b). Then the shock wave focusing occurs in Fig. 6(c), but compared with the case of the $\mathrm{SF}_{6}$ bubble, the shock focusing position is significantly closer to the downstream pole of the R22 bubble, and the shock focusing time is also advanced. As shown in Fig. 6(d), a jet (J) is generated at the right interface of the bubble owing to the high pressure resulting from the shock focusing phenomenon. Subsequently, the reflected shock wave (RS) from the right end wall impinges on the distorted R22 bubble again in Figs. 6(e) and 6(f). Unlike the case of the $\mathrm{SF}_{6}$ bubble, the jet is significantly slender in this case, and the vortices generated on the surfaces of the bubble are also fewer. Although there are some specific differences between the two different cases, due to the values of the Atwood number for the $\mathrm{R}_{2} 2$ and $\mathrm{SF}_{6}$ gases are both larger than zero, namely, the shock-bubble interaction is in a fast/slow (F/S) configuration, ${ }^{13}$ the overall trends of $\mathrm{R} 22$ and $\mathrm{SF}_{6}$ bubbles in the evolution of waves and bubble interface are basically consistent.
When the density of the spherical gas continues to decrease, the density of $\mathrm{Ne}$ is slightly lower than that of the surrounding air, which is characterized by a negative value of Atwood number (At $=-0.19)$. This configuration implies the shock-bubble interaction is in a slow/fast (S/F) configuration. ${ }^{13}$ A sequence of Schlieren pictures showing the interaction between the shock wave and neon bubble is presented in Fig. 7. At the initial stage of the incident shock wave (IS) interacting with the bubble [as shown in Fig. 7(a)], the propagation velocity of the refracted shock wave (TS1) in the bubble is slightly faster than that of the IS outside the bubble because the acoustic impedance of $\mathrm{Ne}$ gas is slightly smaller than that of air. At the same time, it is also found that due to the collision of the IS and the bubble, a reflected rarefaction wave (RRS1) is generated in the vicinity of the left interface of the bubble. As the IS propagates, the RRS1 propagates to the top and the bottom of the flow field continuously, as shown in Fig. 7(b). In Fig. 7(c), the Ne bubble has been completely compressed into a rugby shape, and the IS and transmitted shock wave (TS2) are merging into a straight plane in front of the bubble. It can be seen from Fig. 7(d) that the reflected shock wave (RS) generated by the collision between the IS and the end wall has moved to the right front of the bubble, while the entire Ne bubble has become a half moon shape, and some vortices appear at the top and

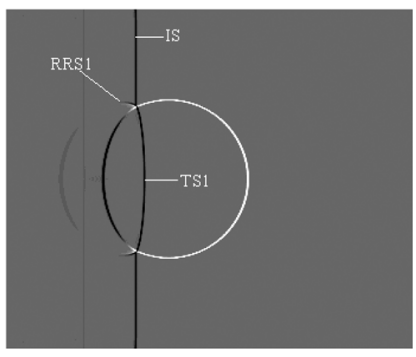

(a) $t=34.2 \mu \mathrm{s}$



(c) $t=139.1 \mu \mathrm{s}$

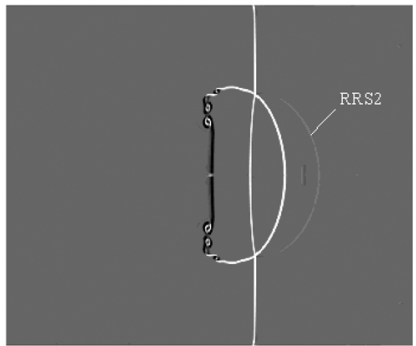

(e) $t=348.1 \mu \mathrm{s}$

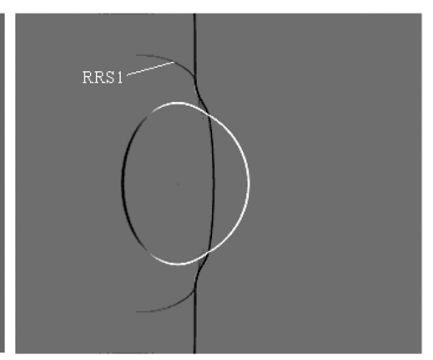

(b) $t=62.8 \mu \mathrm{s}$



(d) $t=300.0 \mu \mathrm{s}$

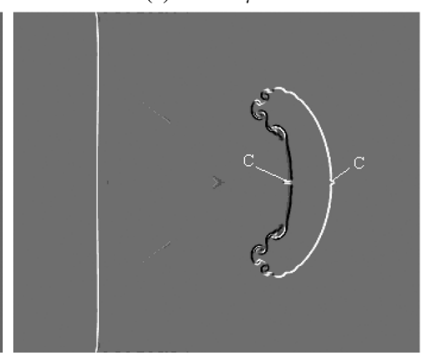

(f) $t=471.7 \mu \mathrm{s}$
FIG. 7. Evolution of the waves and Ne gas bubble. 
the bottom of the left interface. Subsequently, the RS impinges on the distorted bubble again in Fig. 7(e), and another reflected rarefaction wave (RRS2) is generated near the right interface of the bubble. By the time shown in Fig. 7(f), the RS has completely passed through the distorted Ne bubble, and the bubble becomes a shape of a squash. Meanwhile, large vorticities are generated and developed at the top and the bottom of the bubble, and the left and the right interfaces of the bubble generate a very small cusp $(\mathrm{C})$ protruding to the outside of the bubble respectively. As is well known, the baroclinic effect plays a vital role in the production of vorticities. ${ }^{12,15,19,21}$ In the $\mathrm{Ne}$ case, the direction of density gradient is just the opposite to those in the $\mathrm{SF}_{6}$ and $\mathrm{R} 22$ cases; thus, take the upper half of the bubble as an example, the vorticities in Fig. 7 are in the opposite directions compared with those in Figs. 5 and 6.

As a typical light gas used in the RMI study, helium gas is adopted as the fourth gas in our shock-bubble interaction computation. Figure 8 shows the Schlieren pictures of several typical time instants during the interaction between the shock waves and the He bubble. In this configuration (At $=-0.76$ ), the refracted shock wave (TS1) inside the bubble moves much faster than the incident shock wave (IS) outside the bubble in Fig. 8(a), and a reflected rarefaction wave (RRS) is generated when the IS impinges on the



(a) $t=41.2 \mu \mathrm{s}$

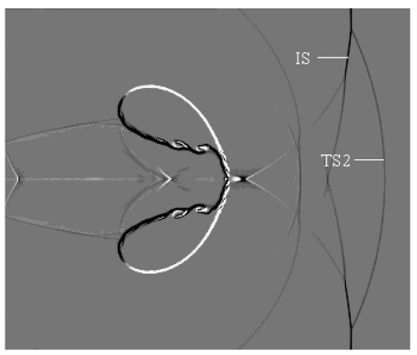

(c) $t=194.7 \mu \mathrm{s}$

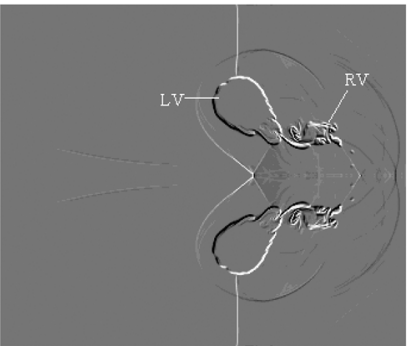

(e) $t=346.6 \mu \mathrm{s}$

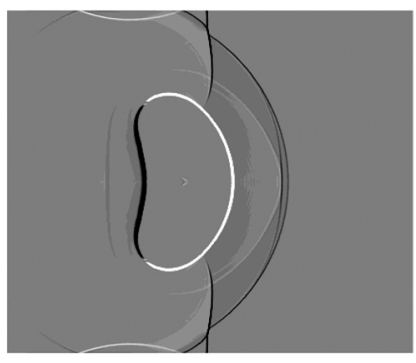

(b) $t=84.8 \mu \mathrm{s}$

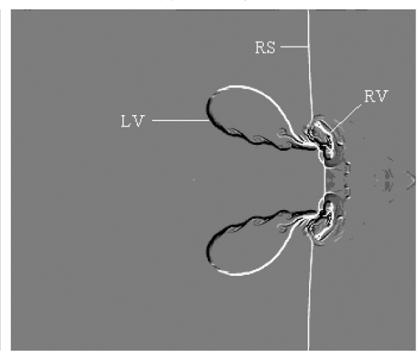

(d) $t=310.8 \mu \mathrm{s}$

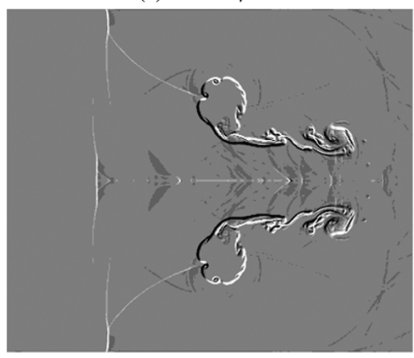

(f) $t=393.5 \mu \mathrm{s}$
FIG. 8. Evolution of the waves and He gas bubble. bubble interface. It is also found that the bubble is flattened in the direction of shock propagation. As time goes by, the left interface of the He bubble begins to sink inward in Fig. 8(b), and the bubble becomes a kidney shape due to the formation of a re-entrant jet at the left interface. . Compared with the outward jet formed in the $\mathrm{SF}_{6}$ and $\mathrm{R} 22$ cases (on the right interface of the bubble), the formation mechanism of inward air jet formed on the left interface of the He bubble is quite different. The density gradient around the light bubble $(\mathrm{He})$ is from the inside to the outside of the bubble, while the density gradient of the heavy bubble ( $\mathrm{SF}_{6}$ and $\left.\mathrm{R} 22\right)$ is in contrast to that of the light bubble. When the shock wave interacts with the bubble, the shock wave induces an upstream-directed pressure gradient. Simultaneously, under the dual actions of density gradient and pressure gradient, vorticities rotating counterclockwise are generated at the upper half of the light bubble, while a large number of vorticities rotating clockwise are generated at the lower half of the light bubble. Furthermore, these vorticities drive the surrounding air to impact the left interface of the He bubble and eventually form a re-entrant jet. However, the direction of the vorticities generated on the upper half and the lower half of the heavy bubble is opposite to that of the light bubble, respectively, so that the heavy bubble cannot generate a re-entrant jet at the left interface of the bubble. While in Fig. 8(c), the jet develops further and eventually impinges on the right interface of the He bubble, which also results in the production of vorticities. Simultaneously, the IS and incident transmitted shock wave (TS2) begin to merge. Subsequently, the distorted $\mathrm{He}$ bubble develops into the vortex rings [left vortex ring (LV) and right vortex ring (RV)] with different rotation direction in Fig. 8(d), and the reflected shock wave (RS) from the right end wall has passed through the RV. When the RS further impinges on the LV, the size of LV obviously decreases in Fig. 8(e); however, LV and RV are still attached to each other. At $t=393.5 \mu \mathrm{s}$ [Fig. 8(f)], the RV is further separated from the LV due to the different rotational direction, and the streamwise stretching of the distorted He bubble interface intensifies the mixing of the air and He gases; thus, the whole flow field shows more turbulent eventually.

\section{Integral diagnostics}

In order to facilitate the study of the evolution of bubbles with time, the time periods during which shock waves interact with bubbles are shown in Table III.

As can be found from Table III, the time period of an incident shock wave interacting with a bubble is continuously shortened as the Atwood number decreases except for the He bubble case. A reasonable explanation is that the density of the He bubble is much smaller than that of the surrounding air; thus, the He bubble moves the fastest along with the shock wave under the impingement among the four cases, which will increase the time period when the incident shock wave interacts with the bubble to an extent.

During the processes of shock waves interacting with bubbles, the values of the spherical bubble volume change continuously under the impingement of the incident and reflected shock waves. In order to study the evolution of bubbles with different values of the Atwood number, the effective bubble volume $V_{\text {effective }}$ is defined first as follows:

$$
V_{\text {effective }}=\int_{D}(1-X) d V
$$


TABLE III. Time periods of shock waves interacting with bubbles, where ISTP denotes the time period in which incident shock wave interacts with the bubble and RSTP denotes the time period in which reflected shock wave interacts with the bubble.

\begin{tabular}{lcccc}
\hline \hline Shock waves & \multicolumn{1}{c}{$\mathrm{SF}_{6}$} & $\mathrm{R} 22$ & $\mathrm{Ne}$ & $\mathrm{He}$ \\
\hline $\mathrm{ISTP}(\mu \mathrm{s})$ & $11.5-141.3$ & $11.5-129.1$ & $11.5-78.0$ & $11.5-99.8$ \\
$\operatorname{RSTP}(\mu \mathrm{s})$ & $351.3-495.9$ & $325.7-467.8$ & $330.47-368.6$ & $304.2-357.8$ \\
\hline \hline
\end{tabular}

where $D$ represents the entire computation domain, and $X$ is the air volume fraction.

Figure 9 presents the evolution of the dimensionless $V_{\text {effective }} / V_{0}$ with time ( $V_{0}$ is the initial bubble volume) for different values of the Atwood number. It can be found that the values of effective volume of the gas bubble for different values of Atwood number basically decrease with time and gradually stabilize after the reflected shock wave passes through the distorted bubble. Especially for the period of the incident and reflected shock waves impinge on the bubble, the values of $V_{\text {effective }} / V_{0}$ decrease greatly. For the cases of At $=0.67$ or 0.51 , the values of $V_{\text {effective }} / V_{0}$ increase slightly behind the incident shock wave, and a probable explanation is that the outward jet formation of $\mathrm{SF}_{6}$ and $\mathrm{R} 22$ bubbles could increase their effective bubble volumes. For the He bubble case $($ At $=-0.76)$, a continuous decline of the effective volume can be found and its value is also the smallest among the four cases. In general, the values of $V_{\text {effective }} / V_{0}$ basically decrease when the Atwood number also decreases. More importantly, the discrepancy among the values of $V_{\text {effective }} / V_{0}$ becomes more obvious when At $<0$.

Mixing between the different fluids inside and outside of the gas bubble is an important aspect of shock-bubble interaction. Considering this, a dimensionless quantity $\xi$ is proposed to scale the mixedness

$$
\xi=\int_{B} X d V / \int_{B} d V
$$

where $B$ represents the bubble region (defined as $Y \leq 0.99$ ). The evolution of the mixedness is plotted in Fig. 10. It is obvious that the values of $\xi$ increase with time in all the four cases, which imply that more and more air has been entrained into the distorted bubbles. However, the growth rate of $\xi$ is relatively slow during the period of the incident shock wave interacting with the bubble, while the



FIG. 9. Evolution of the effective bubble volume. growth rate of $\xi$ rises rapidly after the reflected shock wave passes through the distorted bubble. It should also be noted that for the fast/slow configuration [such as the $\mathrm{SF}_{6}(\mathrm{At}=0.67)$ and $\mathrm{R} 22$ (At $=0.51)$ cases], the values of $\xi$ increase when the Atwood number increases. While for the slow/fast configuration [such as the $\mathrm{Ne}$ (At = $-0.19)$ and $\mathrm{He}(\mathrm{At}=-0.76)$ cases], the values of $\xi$ increase when the Atwood number decreases. In short, heavier or lighter gas bubbles than the ambient air could induce more intense mixedness under the incident and reflected shock conditions. Thus, it can be concluded that the evolution of $\xi$ is not a simply monotonous relationship with the Atwood number.

Considering the vorticities on the bubble interface can greatly affect the mixing of gases inside and outside the bubble, Fig. 11 shows the evolution of the average vorticity with time in the bubble region, and the average vorticity is defined as follows:

$$
\bar{\omega}=\frac{\int_{B}|\omega| d V}{\int_{B} d V} .
$$

For the fast/slow configuration [such as the $\mathrm{SF}_{6}(\mathrm{At}=0.67)$ and R22 (At $=0.51)$ cases], trends of the average vorticity are basically the same in Fig. 11. To be specific, the values of average vorticity both increase when the incident and reflected shock waves impinge on the bubbles. Subsequently, the enhanced vorticities promote the mixing of gases inside and outside the gas bubble and thus accelerate the transfer and consumption of vorticity energy, which can weaken the average vorticity intensity in the bubble region gradually. But in general, the values of average vorticity increase when the Atwood number increases for the fast/slow configuration. In the $\mathrm{He}$ bubble case (At $=-0.76$ ), the average vorticity strengthens rapidly at the stage of incident shock wave interacting with the bubble, and then a partial oscillation emerges owing to the impingement of the waves inside the bubble. Even so, the general trend still rises. When the reflected shock wave impinges on the distorted He bubble again,

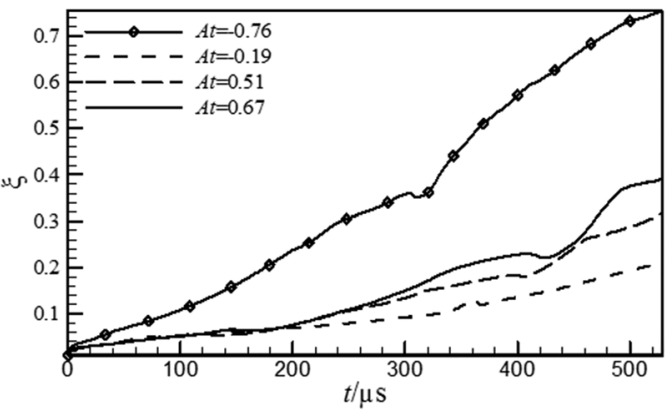

FIG. 10. Evolution of the mixedness. 




FIG. 11. Evolution of the average vorticity.

the average vorticity increases with a higher rate, and then oscillates under the multiinfluences of the impingement of the waves inside the bubble (strengthen the vorticity in the bubble region) and the transfer and consumption of vorticity energy (weaken the vorticity in the bubble region). Besides, it is easy to find that the value of the average vorticity in the Ne bubble case (At $=-0.19$ ) is the smallest among the four cases, which basically remains a small oscillation after $100 \mu \mathrm{s}$. Hence, the average vorticity increases when the Atwood number decreases for the slow/fast configuration. Furthermore, compared with Figs. 10 and 11, it is not hard to find that the evolution trends of the mixedness and the average vorticity are consistent. In other word, a higher average vorticity of the distorted bubble means a higher mixedness of the gases inside and outside the bubble.

The intensity of the vorticity can be expressed by the circulation. In order to further study the vorticity characteristics in the bubble region, the evolution of circulation in the bubble region with time is investigated in Fig. 12, and the formula for calculating the circulation is as follows:

$$
\Gamma=\int_{B} \omega d V
$$

where $\Gamma$ is the net circulation of the bubble region. Since the direction of the density gradient on the bubble interface with At $>0$ is completely opposite to that on the bubble interface with At $<0$, the rotational direction of vorticity is completely opposite in the two different conditions and thus the circulation is positive or negative depending on the positive or negative vorticity in the different



FIG. 12. Evolution of the circulation.
Atwood number cases. From Fig. 12, it is easy to find that the absolute value of circulation of the Ne bubble $(\mathrm{At}=-0.19)$ is the smallest among the four cases, which remains close to zero despite some small oscillations. For the same slow/fast configuration, the circulation value of the He bubble ( $\mathrm{At}=-0.76$ ) is always the largest among the four cases and oscillates around $5 \mathrm{~m}^{2} \mathrm{~s}^{-1}$ after the incident shock wave interacting with the bubble. While for the fast/slow configuration, the trends of the circulation of $\mathrm{SF}_{6}$ bubble $(\mathrm{At}=0.67)$ and $\mathrm{R} 22$ bubble $(\mathrm{At}=0.51)$ are basically the same. The absolute values of circulation of the two cases both increase rapidly during the period of the incident shock waves interacting with bubbles and continue to grow with a small rate. Then, they decrease rapidly after the interaction between the reflected shock waves and bubbles. Although the circulation is affected by the distorted bubble volume and the vorticity simultaneously, the whole relative relations of the absolute values of circulation are in accordance with those in the evolution of the average vorticity in Fig. 11, which indicates that the vorticity dominates the evolution of circulation in the inert shock-bubble interaction process.

\section{CONCLUSIONS}

In the present paper, a detailed numerical investigation of the interaction between a planar shock and a spherical gas bubble containing either $\mathrm{SF}_{6}, \mathrm{R} 22$, $\mathrm{Ne}$, or $\mathrm{He}$ is conducted. High-order numerical schemes and a high grid resolution are utilized. The influences of the Atwood number on the evolution of the shock wave and the gas bubble are clarified. It is shown that the difference in the physical properties between the ambient air and the gas bubble has a significant influence on the evolution of wave pattern and bubble deformation. To be specific, the main conclusions are summarized as follows:

(1) For the fast/slow configuration (At $>0$ ) in the present study (At $=0.67$ and 0.51), the incident shock focuses near the interior right interface of the heavy bubble to form a local high pressure zone, which induces the outward jet subsequently. Besides, the shock focusing position is closer to the downstream pole of the bubble and the shock focusing time is also advanced when the Atwood number decreases.

(2) For the slow/fast configuration (At $<0$ ), the light bubble is easier to be compressed, and the direction of the density gradient on the bubble interface becomes the opposite. Hence, the rotational directions of the vorticities formed at the same position are also reversed compared with those in the fast/slow configuration, and an inward air jet is formed which can impact on the gas bubble from the outside. Especially in the He bubble case, the He gas bubble is divided into upper and lower parts by the re-entrant air jet, and two pairs of vortex rings are generated after the impingement of the reflected shock wave.

(3) From the viewpoint of quantitative analysis, the effective volume of the gas bubble basically decreases when the Atwood number decreases. By contrast, the values of mixedness and average vorticity and the absolute value of circulation all decrease as the Atwood number decreases only for the fast/slow configuration (At $>0$ ), and the trends are reversed for the fast/slow configuration (At $<0$ ). 


\section{ACKNOWLEDGMENTS}

This work was supported by the National Natural Science Foundation of China (Grant Nos. 11872193, 11402102, and 91641113), the Natural Science Foundation of Jiangsu Province of China (Grant No. BK20140524), the Jiangsu Overseas Visiting Scholar Program for University Prominent Young and Middle-aged Teachers and Presidents, and the Youth Talent Cultivation Plan of Jiangsu University.

\section{REFERENCES}

${ }^{1}$ E. S. Oran and V. N. Gamezo, "Origins of the deflagration-to-detonation transition in gas-phase combustion," Combust. Flame 148, 4-47 (2007).

${ }^{2}$ H. H. Teng, Z. L. Jiang, and H. D. Ng, "Numerical study on unstable surfaces of oblique detonations," J. Fluid Mech. 774, 111-128 (2014).

${ }^{3} \mathrm{H}$. Teng, H. D. Ng, and Z. Jiang, "Initiation characteristics of wedge-induced oblique detonation wave in a stoichiometric hydrogen-air mixture," Proc. Combust. Inst. 36(2), 2735-2742 (2017)

${ }^{4}$ J. F. Haas and B. Sturtevant, "Interaction of weak shock waves with cylindrical and spherical gas inhomogeneities," J. Fluid Mech. 181, 41-76 (1987).

${ }^{5}$ J. W. Jacobs, "Shock-induced mixing of a light-gas cylinder," J. Fluid Mech. 234, 629 (1992).

${ }^{6} \mathrm{~J}$. W. Jacobs, "The dynamics of shock accelerated light and heavy gas cylinders," Phys. Fluids A 5, 2239 (1993).

${ }^{7}$ G. Layes, G. Jourdan, and L. Houas, "Experimental investigation of the shock wave interaction with a spherical gas inhomogeneity," Phys. Fluids 17, 028103 (2005).

${ }^{8}$ G. Layes and O. L. Metayer, "Quantitative numerical and experimental studies of the shock accelerated heterogeneous bubbles motion," Phys. Fluids 19, 042105 (2007).

${ }^{9}$ G. Layes, G. Jourdan, and L. Houas, "Experimental study on a plane shock wave accelerating a gas bubble," Phys. Fluids 21, 074102 (2009).

${ }^{10}$ D. Ranjan, J. H. J. Niederhaus, J. G. Oakley, M. H. Anderson, R. Bonazza, and J. A. Greenough, "Shock-bubble interactions: Features of divergent shockrefraction geometry observed in experiments and simulations," Phys. Fluids 20, 036101 (2008)
${ }^{11}$ N. Haehn, C. Weber, J. Oakley, M. Anderson, D. Ranjan, and R. Bonazza, "Experimental study of the shock-bubble interaction with reshock," Shock Waves 22(1), 47-56 (2012).

${ }^{12}$ T. Si, Z. G. Zhai, J. M. Yang, and X. S. Luo, "Experimental investigation of reshocked spherical gas interfaces," Phys. Fluids 24(5), 054101 (2012).

${ }^{13}$ N. J. Zabusky and S. M. Zeng, "Shock cavity implosion morphologies and vortical projectile generation in axisymmetric shock-spherical fast/slow bubble interactions," J. Fluid Mech. 362, 327-346 (1998).

${ }^{14}$ A. Bagabir and D. Drikakis, "Mach number effects on shock-bubble interaction," Shock Waves 11(3), 209-218 (2001).

${ }^{15}$ J. H. J. Niederhaus, J. A. Greenough, J. G. Oakley, D. Ranjan, M. H. Anderson, and R. Bonazza, "A computational parameter study for the three-dimensional shock-bubble interaction," J. Fluid Mech. 594, 84-124 (2008).

${ }^{16}$ Z. G. Zhai, T. Si, X. S. Luo, and J. M. Yang, "On the evolution of spherical gas interfaces accelerated by a planar shock wave," Phys. Fluids 23(8), 084104 (2011). ${ }^{17}$ L. Y. Zou, Z. G. Zhai, J. H. Liu, Y. P. Wang, and C. L. Liu, "Energy convergence effect and jet phenomenon of shock-heavy spherical bubble interaction," Sci. China: Phys., Mech. Astron. 58(12), 124703 (2015).

${ }^{18}$ P. Y. Georgievskiy, V. A. Levin, and O. G. Sutyrin, "Interaction of a shock with elliptical gas bubbles," Shock Waves 25(4), 357-369 (2015).

${ }^{19}$ Y. J. Zhu, G. Dong, B. C. Fan, and Y. X. Liu, "Formation and evolution of vortex rings induced by interactions between shock waves and a low-density bubble," Shock Waves 22, 495-509 (2012).

${ }^{20}$ Y. J. Zhu, L. Yu, J. F. Pan, Z. H. Pan, and P. G. Zhang, "Jet formation of $\mathrm{SF}_{6}$ bubble induced by incident and reflected shock waves," Phys. Fluids 29, 126105 (2017).

${ }^{21}$ Y. J. Zhu, Z. W. Yang, Z. H. Pan, P. G. Zhang, and J. F. Pan, "Numerical investigation of shock-SF 6 bubble interaction with different mach numbers," Comput. Fluids 177, 78-86 (2018).

${ }^{22} \mathrm{D}$. Igra and O. Igra, "Numerical investigation of the interaction between a planar shock wave with square and triangular bubbles containing different gases," Phys. Fluids 30, 056104 (2018).

${ }^{23}$ G. S. Jiang and C. W. Shu, "Efficient implementation of weighted ENO schemes," J. Comput. Phys. 126, 202-228 (1996).

${ }^{24}$ F. Diegelmann, S. Hickel, and N. A. Adams, "Shock mach number influence on reaction wave types and mixing in reactive shock-bubble interaction," Combust. Flame 174, 85-99 (2016). 